\title{
Continuous and Piecewise Affine Lyapunov Functions using the Yoshizawa Construction
}

\author{
Sigurður Hafstein ${ }^{\dagger}$, Christopher M. Kellett ${ }^{\ddagger}$, Huijuan Li $^{*}$
}

\begin{abstract}
We present a novel numerical technique for the computation of a Lyapunov function for nonlinear systems with an asymptotically stable equilibrium point. Our proposed approach constructs a continuous piecewise affine (CPA) function given a suitable partition of the state space, called a triangulation, and values at the vertices of the triangulation. The vertex values are obtained from a Lyapunov function in a classical converse Lyapunov theorem and verification that the obtained CPA function is a Lyapunov function is shown to be equivalent to verification of several simple inequalities. Furthermore, by refining the triangulation, we show that it is always possible to construct a CPA Lyapunov function. Numerical examples are presented demonstrating the effectiveness of the proposed method.
\end{abstract}

\section{INTRODUCTION}

Lyapunov's Second or Direct Method [13] (see also [18]) has proved to be one of the most useful tools for demonstrating stability properties. This is largely due to the fact that if one has a Lyapunov function at hand there is no need to explicitly generate system solutions in order to determine stability. Unfortunately, this frequently trades the difficult problem of generating system solutions for the equally difficult problem of constructing a Lyapunov function. Nonetheless, it is frequently worthwhile numerically searching for a Lyapunov function as it can be used for purposes other than stability analysis such as in estimating a basin of attraction (in the case of local stability) or for inferring robustness properties [11] [20].

So-called converse Lyapunov theorems provide existence results for Lyapunov functions; i.e., assuming a particular stability property holds then there exists an appropriate Lyapunov function. However, such results are largely not constructive in nature and, in fact, depend explicitly on solutions of the system under study. As a consequence, various approaches have been proposed for the numerical construction of Lyapunov functions such as collocation methods [4], [8], graph theoretic methods [2], [9], and semidefinite optimization for sum-of-squares polynomials (known as the SOS method) [16], [17].

$\dagger$ S. Hafstein is with the School of Science and Engineering at Reykjavik University, Iceland sigurdurheru.is. $\ddagger$ C.M. Kellett is with the School of Electrical Engineering and Computer Science, University of Newcastle, Callaghan, New South Wales, Australia Chris.Kellett@newcastle.edu.au. Kellett is supported by ARC Future Fellowship FT1101000746 and by the Alexander von Humboldt Foundation. ${ }^{*} \mathrm{H} . \mathrm{Li}$ is with the Mathematical Institute, University of Bayreuth, 95440 Bayreuth, Germany Huijuan.Li@uni-bayreuth.de. Li is supported by the EU Initial Training Network "Sensitivity Analysis for Deterministic Controller Design - SADCO".
In this paper we consider systems described by ordinary differential equations

$$
\dot{x}=f(x), \quad x \in \mathbb{R}^{n},
$$

where we assume $f(\cdot)$ is twice continuously differentiable, and denote solutions to (1) by $\phi: \mathbb{R}_{\geq 0} \times \mathbb{R}^{n} \rightarrow \mathbb{R}^{n}$. One proposed approach to constructing a Lyapunov function for (1) involves partitioning the state-space into simplices (called a triangulation), defining values for the vertices of every simplex, and for every simplex taking the convex interpolation of those values. This yields a continuous and piecewise affine (CPA) function. If the values at the vertices satisfy a system-dependent set of linear inequalities, then the resulting CPA function is a Lyapunov function.

An approach using linear programming to compute feasible values for the CPA function at the simplex vertices was proposed in [14] with refinements in [6], [1], [5]. However, since linear programming is not very efficient, the question remains if the values at the vertices cannot be fixed by more efficient means with a subsequent speedy test of the validity of the linear inequalities. In this paper, we propose using a construction from a particular converse Lyapunov theorem. Classical converse Lyapunov theorems such as those developed by Massera [15] and Kurzweil [12] rely on integrating solutions from the initial time to infinity. However, Yoshizawa [21] provided an alternate construction that involves taking the supremum over time of the norm of the solution. Initially this appears to provide no improvement towards a constructive approach, but it can be shown that this supremum is actually a maximum over a finite-time horizon. Furthermore, in many cases, this horizon may not be overly long.

We therefore propose a method for constructing CPA Lyapunov functions based on using Yoshizawa's construction for the values at the simplex vertices and subsequently verifying that this yields a true Lyapunov function by checking the validity of the linear inequalities from Theorem 2 below. In Theorem 3 below we demonstrate that this construction will always succeed if the CPA function has enough structure, i.e. the triangulation has a sufficient number of vertices, and the numerical integration of the solution trajectories delivers reliable values.

The paper is organized as follows: in Section II we describe the construction of CPA functions on a given triangulation and the linear program used to verify if a given CPA function is, in fact, a Lyapunov function. In Section III we describe the Lyapunov function construction due to Yoshizawa and 
describe the form of the stability estimates required. In Section IV we present three representative numerical examples and in Section $\mathrm{V}$ we conclude and provide some indications of future work.

\section{Continuous And Piecewise AfFine Lyapunov FUNCTIONS}

In the sequel, we will define continuous and piecewise affine (CPA) functions on suitable triangulations. For a set $\Omega \subset \mathbb{R}^{n}$, we denote the interior of $\Omega$ by $\Omega^{\circ}$ and the closure of $\Omega$ by $\bar{\Omega}$.

Definition 1: We call a finite collection $\mathcal{T}=$ $\left\{\mathfrak{S}_{1}, \mathfrak{S}_{2}, \ldots, \mathfrak{S}_{N}\right\}$ of $n$-simplices in $\mathbb{R}^{n}$ a suitable triangulation if

i) $\mathfrak{S}_{\nu}, \mathfrak{S}_{\mu} \in \mathcal{T}, \nu \neq \mu$, intersect in a common face or not at all.

ii) With $\mathcal{D}_{\mathcal{T}} \doteq \cup_{\nu} \mathfrak{S}_{\nu}, \mathcal{D}_{\mathcal{T}}^{\circ}$ is a connected neighborhood of the origin.

iii) If $0 \in \mathfrak{S}_{\nu}$, then 0 is a vertex of $\mathfrak{S}_{\nu}$.

Remark 1: Property i), often called shape regularity in the theory of FEM, is needed so that we can parameterize every continuous function, affine on every simplex, by specifying its values at the vertices, cf. Remark 2. Property ii) ensures that $\mathcal{D}_{\mathcal{T}}$ is a natural domain for a Lyapunov function and, without Property iii), a function affine on each of the simplices could not have a local minimum at the origin.

For a given suitable triangulation, $\mathcal{T}$, and with $\mathcal{D}_{\mathcal{T}} \doteq$ $\cup_{\mathfrak{S} \in \mathcal{T}} \mathfrak{S}$, we denote the set of all continuous functions $f: \mathcal{D}_{\mathcal{T}} \rightarrow \mathbb{R}$ that are affine on every simplex $\mathfrak{S} \in \mathcal{T}$ by $\mathrm{CPA}[\mathcal{T}]$

Remark 2: A function $f \in \operatorname{CPA}[\mathcal{T}]$ is uniquely determined by its values at the vertices of the simplices of $\mathcal{T}$. To see this, let $\mathfrak{S}_{\nu}=\operatorname{co}\left\{x_{0}, x_{1}, \ldots, x_{n}\right\} \in \mathcal{T}$. Every point $x \in \mathfrak{S}_{\nu}$ can be written uniquely as a convex combination of its vertices, $x=\sum_{i=0}^{n} \lambda_{i}^{x} x_{i}, \lambda_{i}^{x} \geq 0$ for all $i=0,1, \ldots, n$, and $\sum_{i=0}^{n} \lambda_{i}^{x}=1$. The value of $f$ at $x$ is given by $f(x)=$ $\sum_{i=0}^{n} \lambda_{i}^{x} f\left(x_{i}\right)$. Additionally, $f$ has a representation on $\mathfrak{S}_{\nu}$ as $f(x)=w^{T}\left(x-x_{0}\right)+a$ for $w \in \mathbb{R}^{n}$ and $a \in \mathbb{R}$. Let $\nabla f_{\nu} \doteq w$. Then, as shown in [5, Remark 9], $\nabla f_{\nu}$ is linear in the values of $f$ at the vertices $x_{0}, x_{1}, \ldots, x_{n}$.

Our subsequent results will be valid on a domain $\mathcal{D} \subset \mathbb{R}^{n}$ minus a fixed arbitrarily small neighborhood of the origin. We define a $\operatorname{CPA}[\mathcal{T}]$ Lyapunov function that accounts for this.

Definition 2: Let $\mathcal{T}$ be a suitable triangulation and let $V \in \mathrm{CPA}[\mathcal{T}]$ be a positive definite function. Define constants

$$
\begin{gathered}
\alpha_{1}^{*} \doteq \inf _{x \in \mathcal{D}_{\mathcal{T}} \backslash\{0\}} \frac{V(x)}{|x|}, \alpha_{2}^{*} \doteq \sup _{x \in \mathcal{D}_{\mathcal{T}} \backslash\{0\}} \frac{V(x)}{|x|}, \\
\text { and } r \doteq \min _{x \in \partial \mathcal{D}_{\mathcal{T}}}|x| . \text { Let } \varepsilon \in \mathbb{R}_{>0} \text { satisfy } \\
0<\varepsilon<r \alpha_{1}^{*} / \alpha_{2}^{*}
\end{gathered}
$$

and define $B_{\varepsilon} \doteq\left\{x \in \mathbb{R}^{n}:|x|<\varepsilon\right\}$. If there is a constant $\alpha_{3}^{*}>0$ such that

$$
\limsup _{h \rightarrow 0+} \frac{V(x+h f(x))-V(x)}{h} \leq-\alpha_{3}^{*}|x|
$$

for all $x \in\left(\mathcal{D}_{\mathcal{T}} \backslash B_{\varepsilon}\right)^{\circ}$ we call $V$ a $\mathrm{CPA}[\mathcal{T}]$ Lyapunov function for (1) on $\mathcal{D}_{\mathcal{T}} \backslash B_{\varepsilon}$.

The implication of a $\mathrm{CPA}[\mathcal{T}]$ Lyapunov function for (1) on $\mathcal{D}_{\mathcal{T}} \backslash B_{\varepsilon}$ is slightly weaker than asymptotic stability.

Theorem 1: Given a suitable triangulation, $\mathcal{T}$, and $\varepsilon \in$ $\mathbb{R}_{>0}$, assume that $V: \mathcal{D} \rightarrow \mathbb{R}_{\geq 0}$ is a $\mathrm{CPA}[\mathcal{T}]$ Lyapunov function for (1) on $\mathcal{D}_{\mathcal{T}} \backslash B_{\varepsilon}$. For every $c \in \mathbb{R}_{\geq 0}$ define the sublevel set $L_{V, c} \doteq\left\{x \in \mathcal{D}_{\mathcal{T}}: V(x) \leq c\right\}$ and let $m \doteq \max _{|x|=\varepsilon} V(x)$ and $M \doteq \min _{x \in \partial \mathcal{D}_{\mathcal{T}}} V(x)$. Then, for every $c \in[m, M)$ we have $B_{\varepsilon} \subset L_{V, c} \subset \mathcal{D}_{\mathcal{T}}^{\circ}$ and there exists $a T_{c} \geq 0$ such that $\phi\left(t, L_{V, c}\right) \subset L_{V, m}$ for all $t \geq T_{c}$.

In other words, a $\mathrm{CPA}[\mathcal{T}]$ Lyapunov function implies asymptotic stability of the set $L_{V, m}$. The proof is similar to [6, Theorem 6.16] and we omit the details due to space constraints.

The following theorem and corollary provide a set of linear inequalities such that, if a given CPA function satisfies the inequalities then it is a CPA Lyapunov function.

Theorem 2: Let $\mathcal{T}$ be a suitable triangulation and let $V \in \mathrm{CPA}[\mathcal{T}]$. Define $\mathfrak{S}_{\nu} \doteq \operatorname{co}\left\{x_{0}^{\nu}, x_{1}^{\nu}, \ldots, x_{n}^{\nu}\right\} \in \mathcal{T}$ and let $B_{\nu} \in \mathbb{R}_{>0}$ satisfy

$$
B_{\nu} \geq \max _{\substack{i, j, k=1,2, \ldots, n \\ x \in \mathfrak{S}_{\nu}}}\left|\frac{\partial^{2} f_{k}}{\partial x_{i} \partial x_{j}}(x)\right| .
$$

For each $\mathfrak{S}_{\nu}$, for $i=0,1, \ldots, n$ define the constants

$$
E_{i, \nu} \doteq \frac{n B_{\nu}}{2}\left|x_{i}-x_{0}\right|\left(\left|x_{i}-x_{0}\right|+\operatorname{diam}\left(\mathfrak{S}_{\nu}\right)\right) .
$$

Then, for every $\mathfrak{S}_{\nu}$ such that the inequalities

$$
0>\nabla V_{\nu} \cdot f\left(x_{i}^{\nu}\right)+\left|\nabla V_{\nu}\right|_{1} E_{i, \nu}
$$

hold for all $i=0,1, \ldots, n$, we have

$$
0>\nabla V_{\nu} \cdot f(x)
$$

for all $x \in \mathfrak{S}_{\nu}$.

Corollary 1: Assume that $V \in \mathrm{CPA}[\mathcal{T}]$ from Theorem 2 is positive definite and that the constant $\varepsilon \in \mathbb{R}_{>0}$ fulfills (2). If the inequalities (3) are fulfilled for all $\mathfrak{S}_{\nu} \in \mathcal{T}$ with $\mathfrak{S}_{\nu} \cap B_{\varepsilon}^{C} \neq \emptyset$, then $V$ is a CPA Lyapunov function for (1) on $\mathcal{D}_{\mathcal{T}} \backslash B_{\varepsilon}$.

The proofs of Theorem 2 and Corollary 1 can be proved in a manner similar to [5, Theorem 1]. We omit the details.

Remark 3: The usefulness of Theorem 2 is that it reduces the verification that a function $V \in \mathrm{CPA}[\mathcal{T}]$ is a Lyapunov function for (1) to the verification of a finite number of inequalities (3). Finding a candidate CPA Lyapunov function can be done as in [1], [5], [6], [14], via linear programming. Alternatively, as in this paper, one can define $V \in \operatorname{CPA}[\mathcal{T}]$ by computing suitable values at the vertices of the simplices of $\mathcal{T}$ and then verify the inequalities (3).

In the next theorem we consider CPA approximations to functions.

Definition 3: Let $\mathcal{D} \subset \mathbb{R}^{n}$ be a domain, $f: \mathcal{D} \rightarrow \mathbb{R}$ be a function, and $\mathcal{T}$ be a triangulation such that $\mathcal{D}_{\mathcal{T}} \subset \mathcal{D}$. The $\mathrm{CPA}[\mathcal{T}]$ approximation $g$ to $f$ on $\mathcal{D}_{\mathcal{T}}$ is the function $g \in \mathrm{CPA}[\mathcal{T}]$ defined by $g(x)=f(x)$ for all vertices $x$ of all simplices in $\mathcal{T}$. 
We additionally need that the simplices in the triangulation $\mathcal{T}$ are not too close to being degenerate. This property can be quantified as follows: For an $n$-simplex $\mathfrak{S}_{\nu} \doteq$ $\operatorname{co}\left\{x_{0}, x_{1}, \ldots, x_{n}\right\} \in \mathcal{T}$ define its shape-matrix as $X_{\nu}$ by writing the vectors $x_{1}-x_{0}, x_{2}-x_{0}, \ldots, x_{n}-x_{0}$ in its rows subsequently. The degeneracy of the simplex $X_{\nu}$ can now be quantified through the value $\operatorname{diam}\left(\mathfrak{S}_{\nu}\right)\left|X_{\nu}^{-1}\right|$, where $\left|X_{\nu}^{-1}\right|$ is the spectral norm of the inverse of $X_{\nu}$ (see part (ii) in the proof of [1, Theorem 4.6]).

Theorem 3: Let $\mathcal{C}, \mathcal{D} \subset \mathbb{R}^{n}$ be simply connected compact neighborhoods of the origin such that $\overline{\mathcal{C}^{\circ}}=\mathcal{C}, \overline{\mathcal{D}^{\circ}}=\mathcal{D}$, and $\mathcal{C} \subset \mathcal{D}^{\circ}$. Assume that $W \in C^{2}(\mathcal{D})$ is a Lyapunov function for (1). Set $r \doteq \min _{x \in \partial \mathcal{C}}|x|$ and let $\varepsilon \in \mathbb{R}_{>0}$ satisfy

$$
\varepsilon<r \cdot \frac{\inf _{x \in \mathcal{D} \backslash\{0\}} W(x) /|x|}{\sup _{x \in \mathcal{D} \backslash\{0\}} W(x) /|x|} .
$$

Then for every $R>0$ there exists a $\delta_{R}>0$ such that, for any triangulation $\mathcal{T}$ satisfying

1) $\mathcal{C} \subset \mathcal{D}_{\mathcal{T}} \subset \mathcal{D}$

2) $\max _{\mathfrak{S}_{\nu} \in \mathcal{T}} \operatorname{diam}\left(\mathfrak{S}_{\nu}\right) \leq \delta_{R}$, and

3) $\max _{\mathfrak{S}_{\nu} \in \mathcal{T}} \operatorname{diam}\left(\mathfrak{S}_{\nu}\right)\left|X_{\nu}^{-1}\right| \leq R$

the $\mathrm{CPA}[\mathcal{T}]$ approximation $V$ to $W$ on $\mathcal{D}_{\mathcal{T}}$ is a CPA Lyapunov function for (1) on $\mathcal{D}_{\mathcal{T}} \backslash B_{\varepsilon}$.

The proof of Theorem 3 follows [5, Theorem 5] and we omit the details due to space constraints.

Remark 4: Theorem 3 is more constructive than it might seem at first glance since a given triangulation $\mathcal{T}$ can be manipulated to deliver a new triangulation $\mathcal{T}^{*}$ with smaller simplices without increasing their degeneracy. As a consequence, it is always possible to find a triangulation that admits a CPA Lyapunov function approximating a twice continuously differentiable Lyapunov function.

\section{YoshizAwA CONSTRUCTION OF LYAPUNOV FUNCTIONS}

We now turn to the question of how to define the vertex values of each simplex in order to obtain a CPA Lyapunov function. We propose using a numerical approximation of a construction initially proposed by Yoshizawa in proving a converse Lyapunov theorem [21]. We make use of the standard function classes $\mathcal{K}_{\infty}$ and $\mathcal{K} \mathcal{L}$ (see [7], [10]).

Let the open set $\mathcal{D} \subset \mathbb{R}^{n}$ be such that the origin is contained in $\mathcal{D}$. Suppose (1) is $\mathcal{K} \mathcal{L}$-stable on $\mathcal{D}$; i.e., there exists $\beta \in \mathcal{K} \mathcal{L}$ so that

$$
|\phi(t, x)| \leq \beta(|x|, t), \quad \forall x \in \mathcal{D}, t \in \mathbb{R}_{\geq 0} .
$$

It was shown in [20, Proposition 1] that $\mathcal{K} \mathcal{L}$-stability is equivalent to (local) asymptotic stability of the origin for (1) where $\mathcal{D}$ is contained in the basin of attraction. When $\mathcal{D}=\mathbb{R}^{n}, \mathcal{K} \mathcal{L}$-stability is equivalent to global asymptotic stability of the origin for (1). We will refer to the function $\beta \in \mathcal{K} \mathcal{L}$ of (4) as a stability estimate.

In what follows we will make use of Sontag's lemma on $\mathcal{K} \mathcal{L}$-estimates [19, Proposition 7] ([10, Lemma 7]):

Lemma 1: Given $\beta \in \mathcal{K} \mathcal{L}$ and $\lambda \in \mathbb{R}_{>0}$, there exist $\alpha_{1}, \alpha_{2} \in \mathcal{K}_{\infty}$ so that, for all $s, t \in \mathbb{R}_{\geq 0}$

$$
\alpha_{1}(\beta(s, t)) \leq \alpha_{2}(s) e^{-\lambda t} .
$$

Definition 4: Given a stability estimate $\beta \in \mathcal{K} \mathcal{L}$, let $\alpha_{1}, \alpha_{2} \in \mathcal{K}_{\infty}$ come from Lemma 1 with $\lambda=2$. We call the function $V: \mathbb{R}^{n} \rightarrow \mathbb{R}_{\geq 0}$ defined by

$$
V(x) \doteq \sup _{t \geq 0} \alpha_{1}(|\phi(t, x)|) e^{t}
$$

a Yoshizawa-Lyapunov function.

As shown in [20] the Yoshizawa-Lyapunov function satisfies the bounds

$$
\alpha_{1}(|x|) \leq V(x) \leq \alpha_{2}(|x|)
$$

and the decrease condition

$$
V(\phi(t, x)) \leq V(x) e^{-t}
$$

The lower bound in (6) is trivial by considering $t=0$ while the upper bound follows from

$$
\begin{aligned}
V(x) & \leq \sup _{t \geq 0} \alpha_{1}(\beta(|x|, t)) e^{t} \\
& \leq \alpha_{2}(|x|) e^{-2 t+t} \leq \alpha_{2}(|x|)
\end{aligned}
$$

As shown in [20], when the vector field of (1) is locally Lipschitz the function $V(\cdot)$ is continuous (except possibly at the origin) and there exists a time $T(x)$ so that

$$
V(x)=\max _{t \in[0, T(x)]} \alpha_{1}(|\phi(t, x)|) e^{t} .
$$

We can calculate the time $T(x)$ explicitly as [20, Claim 2]

$$
T(x)=-\ln \left(\frac{V(x)}{\alpha_{2}(|x|)}\right)+1, \quad x \neq 0
$$

and with the upper and lower bounds on $V$ we see that

$$
\begin{aligned}
0 \leq T(x) & \leq-\ln \left(\frac{\alpha_{1}(|x|)}{\alpha_{2}(|x|)}\right)+1 \\
& =\ln \left(\frac{\alpha_{2}(|x|)}{\alpha_{1}(|x|)}\right)+1
\end{aligned}
$$

Recall that our intention is to calculate $V(x)$ for each $x$ that is a simplex vertex. In order to do this, we clearly need a solution to (1) from each such $x$. As a closed form solution is generally not available, we will resort to numerical integration in order to calculate $V(x)$ given by (5). For this approach to be numerically tractable, we require that the time horizon $T(x)$ given by (11) not be too large. We present two examples of stability estimates and derive $T(x)$ in each case.

\section{A. Example 1 - Exponentially Stable Estimates}

Suppose the stability estimate is given by

$$
|\phi(t, x)| \leq \alpha(|x|) e^{-\mu t}, \quad \mu>0,
$$

where $\alpha(s) \geq s$. Then, for all $s \in \mathbb{R}_{\geq 0}$, we can define

$$
\alpha_{1}(s) \doteq s^{2 / \mu}, \quad \text { and } \quad \alpha_{2}(s) \doteq(\alpha(s))^{2 / \mu}
$$

so that, for all $x \in \mathbb{R}^{n}$ and $t \in \mathbb{R}_{\geq 0}$,

$$
\alpha_{1}\left(\alpha(|x|) e^{-\mu t}\right) \leq(\alpha(|x|))^{2 / \mu} e^{-2 t}=\alpha_{2}(|x|) e^{-2 t} .
$$


We therefore see that, in this case, an upper bound for the time horizon to optimize over is given by

$$
T(x) \leq \frac{2}{\mu} \ln \left(\frac{\alpha(|x|)}{|x|}\right)+1
$$

where the assumption that $\alpha(s) \geq s$ for all $s \in \mathbb{R}_{\geq 0}$ guarantees that $T(x) \geq 1$.

If $\alpha(s)=M s$ for some $M>1$, then an upper bound for the time horizon necessary to optimize over is independent of the point $x$ and is given by

$$
T(x)=T=-\ln M^{-2 / \mu}+1=\frac{2}{\mu} \ln M+1 .
$$

In Section IV-A this bound will be used to compute values of the Yoshizawa-Lyapunov function for a linear system.

\section{B. Example 2}

With the functions $\alpha_{1}, \alpha_{2} \in \mathcal{K}_{\infty}$ given by

$$
\alpha_{1}^{-1}(s) \doteq e^{s}-1, \quad \alpha_{2}(s)=M s
$$

we capture functions $\beta \in \mathcal{K} \mathcal{L}$ satisfying

$$
\beta(s, t) \leq \exp \left(M s e^{-2 t}\right)-1
$$

and the optimization horizon bound is given by

$$
T(x) \leq \ln \left(\frac{M|x|}{\ln (1+|x|)}\right)+1 .
$$

The horizon length grows with increasing $|x|$ but not too quickly. For example, with $M=10:|x|=1$ yields $T(x)=$ 3.67 and $|x|=100$ yields $T(x)=6.38$.

Remark 5: There are two difficulties we encounter in trying to calculate (5). The first difficulty lies with finding a stability estimate $\beta \in \mathcal{K} \mathcal{L}$ or even with verifying that a particular stability estimate such as (13) holds for a particular system (1). There seems to be little that can be done to circumvent this problem.

The second difficulty is that Sontag's lemma on $\mathcal{K} \mathcal{L}$ estimates is not constructive and, to the best of the authors' knowledge, given an arbitrary $\beta \in \mathcal{K} \mathcal{L}$, there are currently no constructive techniques for finding $\alpha_{1}, \alpha_{2} \in \mathcal{K}_{\infty}$.

Remark 6: As previously mentioned, in [20] it was demonstrated that the Yoshizawa-Lyapunov function (5) is continuous except possibly at the origin. However, in order to make use of Theorem 3 we would clearly prefer that the Yoshizawa-Lyapunov function be twice continuously differentiable. Standard smoothing techniques can be applied to the Yoshizawa-Lyapunov function to obtain a smooth function, however these techniques do not generally provide us with an easily calculable function such as (5). Rather, we would prefer to demonstrate directly that the YoshizawaLyapunov function inherits the regularity of the vector field of (1). It is known that, in general, there exists a Lyapunov function that inherits the regularity property of the vector field defining (1) (see [3]), however, it remains to be shown that that the Yoshizawa-Lyapunov function (5) inherits the regularity property of the vector field. This is a subject of future work.

\section{NUMERICAL EXAMPLES}

While the Yoshizawa construction (9) provides an exact value for a Lyapunov function at each point, this assumes we have an exact solution of the differential equation. In this section we present three numerical examples of CPA Lyapunov functions. In each case, we first define a triangulation on a region of the state space that includes the origin in its interior. For technical reasons, we excise a small neighborhood of the origin.

For each example we calculate a stability estimate and, with the triangulation defined, we then use a simple Euler integration scheme to approximate the values of the Yoshizawa-Lyapunov function (9) at all vertices of all simplices. We then check the inequalities (3) to verify that the function defined by taking the convex interpolation on each simplex of the Yoshizawa-Lyapunov function values is, in fact, a CPA-Lyapunov function.

\section{A. Example 3 - Linear System}

Consider the linear system

$$
\dot{x}=A x=\left[\begin{array}{cc}
1 & 1 \\
-5 & -3
\end{array}\right] \text {. }
$$

We observe that the origin is globally exponentially stable as the eigenvalues are at $-1 \pm i$ and, by solving the Lyapunov equation $A^{T} P+P A=-\mathrm{Id}$, a Lyapunov function is given by

$$
V(x)=x^{T} P x=x^{T}\left[\begin{array}{cc}
4.5 & 1 \\
1 & 0.5
\end{array}\right] x .
$$

By explicitly calculating the solutions of (14) we see that the system satisfies the stability estimate

$$
|\phi(t, x)| \leq 7|x| e^{-t}, \quad \forall x \in \mathbb{R}^{2}, t \in \mathbb{R}_{\geq 0} .
$$

From Section III-A, with $\alpha(s)=7 s$ and $\mu=1$ we see that $\alpha_{1}(s)=s^{2}, \alpha_{2}(s)=49 s^{2}$, and $T(x)=T=5$. According to the above proposed procedure, we define a triangulation and define the values at the simplex vertices by approximating (5) to obtain a continuous and piecewise affine function $V_{1}(x)$ for (14) as shown in Figure 1. The neighborhood of the origin $\left\{x \in \mathbb{R}^{2}:|x|<0.048\right\}$, is excluded. It is straightforward to numerically verify the inequalities (3) to conclude that the obtained function, $V_{1}(x)$, is in fact a CPA-Lyapunov function.

The function $V(x)$ given by (15) has a similar though slightly different shape. Level curves for $V_{1}(x)$ are shown in Figure 2 and level curves for $V(x)$ are shown in Figure 3 for comparison.

\section{B. Example 4 - Simple Nonlinear System}

Consider the system

$$
\dot{x}=-x^{3}
$$

which has solution

$$
\phi(t, x)=\frac{x}{\sqrt{1+2 x^{2} t}}, \forall x \in \mathbb{R}, t \in \mathbb{R}_{\geq 0} .
$$






Fig. 1. CPA Lyapunov function $V_{1}(x)$ for system (14).

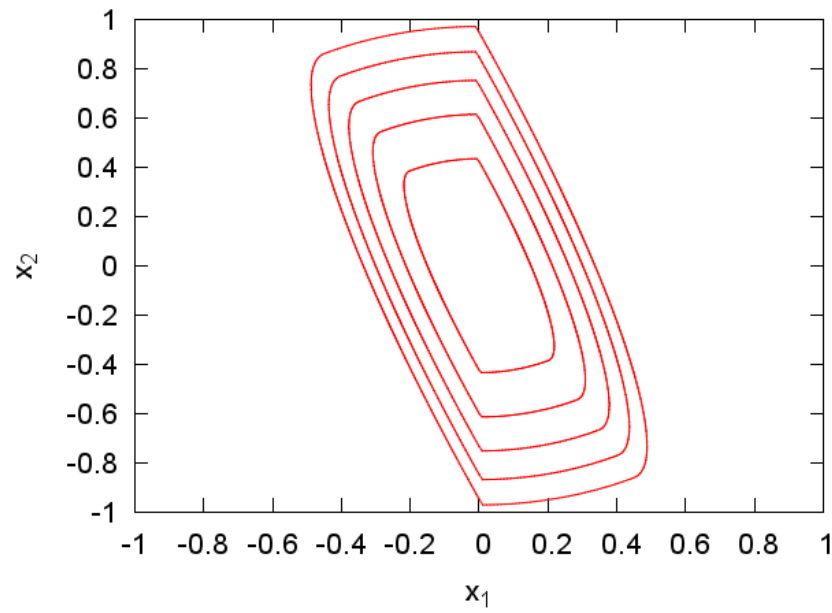

Fig. 2. Level curves of $V_{1}(x)$ for values $0.189,0.378,0.567,0.756$, and 0.945 .



Fig. 3. Level curves of $V(x)$ for values $0.083,0.166$, and 0.249 .

We observe that the norm of the solution is in fact a $\mathcal{K} \mathcal{L}$ function and, consequently, immediately provides a stability estimate. We can verify that the functions

$$
\alpha_{1}(s)=\alpha_{2}(s) \doteq\left\{\begin{array}{cc}
0 & , \quad s=0 \\
s \exp \left(-\frac{1}{s^{2}}\right) & , \quad s>0
\end{array}\right.
$$

are such that

$$
\alpha_{1}\left(\frac{s}{\sqrt{1+2 s^{2} t}}\right) \leq \alpha_{2}(s) e^{-2 t} .
$$

As before, we define a triangulation and calculate the values at the vertices by approximating (5). A convex interpolation of these values on each simplex then yields a CPA function and the inequalities (3) are used to verify that the calculated CPA function is a CPA-Lyapunov function.

We note that, for any $p \in \mathbb{Z}_{\geq 1}$ and $c \in \mathbb{R}_{>0}$, a Lyapunov function for (17) is given by

$$
V(x)=c x^{2 p}, \quad \forall x \in \mathbb{R} .
$$

Figure 4 shows the CPA-Lyapunov function $V_{2}(x)$ for system (17) for $4.8 \geq|x| \geq 0.04332$. For comparison, Figure 4 also shows the known Lyapunov function (21) with $p=2, c=0.01$.



Fig. 4. Lyapunov functions $V(x)=0.01 x^{4}$ (green curve) and $V_{2}(x)$ (red curve) for system (17).

\section{Example 5 - Nonlinear System}

Consider the two-dimensional nonlinear system given by

$$
\begin{aligned}
& \dot{x}_{1}=-x_{2}-\left(1-x_{1}^{2}-x_{2}^{2}\right) x_{1} \\
& \dot{x}_{2}=x_{1}-\left(1-x_{1}^{2}-x_{2}^{2}\right) x_{2} .
\end{aligned}
$$

This system has the unit circle as a periodic orbit and the origin as a locally asymptotically stable equilibrium. On any compact subset of the unit ball, the simple quadratic

$$
V(x) \doteq \frac{1}{2} x_{1}^{2}+\frac{1}{2} x_{2}^{2}
$$

is a known Lyapunov function.

Fix $R \in(0,1)$. Then, for any initial conditions satisfying

$$
x_{1}^{2}+x_{2}^{2} \leq R
$$


we have the stability estimate

$$
|\phi(t, x)| \leq|x| e^{-(1-R) t}
$$

and, from Section III-A, we can calculate

$$
\alpha_{1}(s)=\alpha_{2}(s)=s^{2 /(1-R)}
$$

and $T(x)=T=1$.

For this example,with $R=0.94478$ and using the numerical procedure previously outlined, a CPA-Lyapunov function $V_{3}(x)$ of system (22) was computed and is shown in Figure 5 for $|x| \in[0.012, R]$. For comparison, Figure 5 also shows the known Lyapunov function (23).



Fig. 5. Difference between Lyapunov functions $V(x)$ (upper arrow points) and $V_{3}(x)$ (lower arrow points) for system (22).

\section{Conclusions}

In this paper we have presented a novel technique for the numerical construction of Lyapunov functions given a stability estimate in the form of a $\mathcal{K} \mathcal{L}$-bound on the norm of system trajectories. For a suitable triangulation of the statespace, at each simplex vertex we calculate the value of a Lyapunov function construction due to Yoshizawa [21]. From these values, we then define a CPA function on the domain minus an arbitrarily small neighborhood of the origin. We can verify that the CPA function thus defined is a Lyapunov function (Corollary 1). If the CPA function thus defined is not a Lyapunov function (i.e., does not satisfy (3)), we can refine the triangulation and construct a new CPA function. If the Yoshizawa-Lyapunov function is twice continuously differentiable, then this process of refining the triangulation then yields a CPA Lyapunov function in a finite number of steps (Theorem 3).

\section{REFERENCES}

[1] R. Baier, L. Grüne, and S. Hafstein. Linear programming based Lyapunov function computation for differential inclusions. Discrete and Continuous Dynamical Systems Series B, 17:33-56, 2012.

[2] H. Ban and W. Kalies. A computational approach to Conley's decomposition theorem. Journal of Computational and Nonlinear Dynamics, 1-4:312-319, 2006.

[3] E. A. Barbashin. Existence of smooth solutions of some linear equations with partial derivatives. Doklady Akademii Nauk SSSR, 72(3), 1950.

[4] P. Giesl. Construction of Global Lyapunov Functions Using Radial Basis Functions. Number 1904 in Lecture Notes in Mathematics. Springer, 2007.

[5] P. Giesl and S. Hafstein. Revised CPA method to compute Lyapunov functions for nonlinear systems. Journal of Mathematical Analysis and Applications, 2013 (in press).

[6] S. Hafstein. An Algorithm for Constructing Lyapunov Functions. Electronic Journal of Differential Equations Mongraphs, 2007.

[7] W. Hahn. Stability of Motion. Springer-Verlag, 1967.

[8] T. Johansen. Computation of Lyapunov functions for smooth nonlinear systems using convex optimization. Automatica, 36:1617-1626, 2000.

[9] W. Kalies, K. Mischaikow, and R. VanderVorst. An algorithmic approach to chain recurrence. Foundations of Computational Mathematics, 5-4:409-449, 2005.

[10] C. M. Kellett. A compendium of comparsion function results Submitted, December 2012.

[11] C. M. Kellett and A. R. Teel. On the robustness of $\mathcal{K} \mathcal{L}$-stability for difference inclusions: Smooth discrete-time Lyapunov functions. SIAM Journal on Control and Optimization, 44(3):777-800, 2005.

[12] J. Kurzweil. On the inversion of Ljapunov's second theorem on stability of motion. Chechoslovakian Mathematics Journal, 81:217259, 455-484, 1956. English translation in American Mathematical Society Translations (2), v. 24, pp. 19-77.

[13] A. M. Lyapunov. The general problem of the stability of motion. Math Soc. of Kharkov, 1892. (Russian). (English Translation, International J. of Control, 55:531-773, 1992).

[14] S. Marinosson. Lyapunov function construction for ordinary differential equations with linear programming. Dynamical Systems, 17:137-150, 2002.

[15] J. L. Massera. On Liapounoff's conditions of stability. Annals of Mathematics, 50:705-721, 1949.

[16] A. Papachristodoulou and S. Prajna. The construction of Lyapunov functions using the sum of squares decomposition. In Proceedings of the 41st IEEE Conference on Decision and Control, pages 3482-3487, 2002.

[17] M. Peet and A. Papachristodoulou. A converse sum-of-squares Lyapunov result: An existence proof based on the Picard iteration. In Proceedings of the 49th IEEE Conference on Decision and Control, pages 5949-5954, 2010.

[18] N. Rouche, P. Habets, and M. Laloy. Stability Theory by Liapunov's Direct Method. Springer-Verlag, 1977.

[19] E. D. Sontag. Comments on integral variants of ISS. Systems and Control Letters, 34(1-2):93-100, 1998.

[20] A. R. Teel and L. Praly. A smooth Lyapunov function from a class$\mathcal{K} \mathcal{L}$ estimate involving two positive semidefinite functions. ESAIM Control Optim. Calc. Var., 5:313-367, 2000.

[21] T. Yoshizawa. Stability Theory by Liapunov's Second Method. Mathematical Society of Japan, 1966. 